\title{
Modification of cluster radio halo appearance by the thermal Sunyaev-Zeldovich effect
}

\author{
T. A. Enßlin ${ }^{\star}$ \\ Max-Planck-Institut für Astrophysik, Karl-Schwarzschild-Str.1, Postfach 1317, 85741 Garching, Germany \\ Received 11 October 2002 / Accepted 31 October 2002

\begin{abstract}
We discuss the consequences of the spectral and morphological modification of galaxy cluster radio halos due to the Sunyaev-Zeldovich (SZ) effect for the interpretation of existing and upcoming high frequency radio observations. Likely these modifications have affected the interpretation of the existing Coma cluster radio data. The radio halo emission visible at low $(\$ 5 \mathrm{GHz})$ frequencies is at higher $(>10 \mathrm{GHz})$ frequencies completely over-compensated by the thermal SZ decrement. Thus, the total radio emission of a galaxy cluster goes through zero (in comparison to the constant cosmic microwave background (CMB) emission) at a frequency of several GHz. Since the radio halo brightness has a narrow radial profile compared to the $\mathrm{SZ}$ decrement, a central emission region is surrounded by a decrement within the intermediate frequency range of several GHz. The size of this emission regions shrinks with increasing frequency until the decrement dominates everywhere in the cluster.
\end{abstract}

Key words. intergalactic medium - galaxies: cluster: general - radio continuum: general - radiation mechanism: thermal radiation mechanism: non-thermal - cosmic microwave background

\section{Introduction}

Clusters of galaxies are filled by a hot $\left(k T_{\mathrm{e}} \sim 5 \mathrm{keV}\right)$ plasma. Some - if not all - clusters host ultra-relativistic particle populations, observable by synchrotron emission of $\sim 10$ $\mathrm{GeV}$ electrons. This emission forms the cluster radio halos. Recently, the number of detected cluster radio halos increased significantly (Giovannini et al. 1999; Giovannini \& Feretti 2000; Kempner \& Sarazin 2001) and the prospects for upcoming sensitive radio telescopes to detect large numbers of cluster radio halos are promising (Enßlin \& Röttgering 2002). Radio halos seem to be triggered by strong cluster merger (Buote 2001), although the detailed physical mechanism providing the energetic electrons is still to be revealed. Two classes of theoretical models are discussed in the literature: i) in-situ acceleration of the rapidly cooling electrons by turbulence and shock waves throughout the cluster volume (Jaffe 1977, and others), and ii) secondary electron injection after particle physical interactions. In-situ acceleration typically predicts steepening radio spectra due to the decreasing ratio of acceleration efficiency to radiative losses for higher energy electrons. In order to confirm such models a detailed knowledge of the high frequency radio halo spectrum and morphology is desirable. The classical secondary injection mechanism is the decay of charged pions which were produced in hadronic interaction of relativistic protons with the background gas (Dennison 1980, and others). However, also the self-annihilation of neutralino dark matter particles was proposed as an electron source

\footnotetext{
* e-mail: ensslin@mpa-garching.mpg.de
}

(Colafrancesco \& Mele 2001) although the observed correlation of radio halos with merging events clearly disfavours this scenario. Nevertheless, such dark matter based electron sources may lead to a lower level of radio emission and a search for such emission has the potential to constrain scenarios of the nature of the dark matter (Böhm et al. 2002).

Such desirable high frequency measurements of the cluster halo emission will unavoidably be affected by the thermal SZ effect (Zeldovich \& Sunyaev 1969; Sunyaev \& Zeldovich 1980) of the hot electrons in the cluster plasma, as was also pointed out by Liang et al. (2000). Our goal is to discuss the effects arising from the interference of these two radiation mechanisms.

We use $H_{0}=50 h_{50} \mathrm{~km} \mathrm{~s}^{-1} \mathrm{Mpc}^{-1}$, and assume $H_{0}=$ $65 \mathrm{~km} \mathrm{~s}^{-1} \mathrm{Mpc}^{-1}$ in numerical examples.

\section{Thermal Sunyaev-Zeldovich effect}

The CMB has a perfect blackbody brightness spectrum $I_{\mathrm{cmb}}(v)=i_{0} i(x)$, with $x=h v /\left(k T_{\mathrm{cmb}}\right), i_{0}=2\left(k T_{\mathrm{cmb}}\right)^{3} /(h c)^{2}$, and $i(x)=x^{3} /\left(\mathrm{e}^{x}-1\right)$. The inverse Compton interaction of CMB photons with hot electrons in a galaxy cluster modifies the CMB spectrum. For small optical depth this is well described by

$\delta I_{\mathrm{SZ}}(v)=i_{0} g(x) y$, with $y=\frac{\sigma_{\mathrm{T}}}{m_{\mathrm{e}} c^{2}} \int \mathrm{d} l n_{\mathrm{e}} k T_{\mathrm{e}}$, 


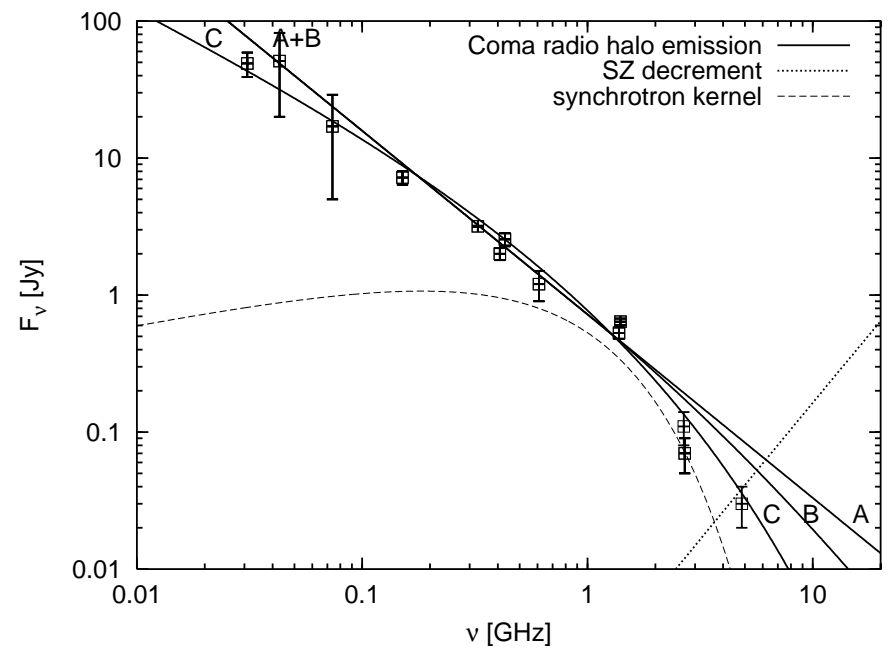

Fig. 1. Observed radio halo fluxes of the Coma cluster as compiled by Thierbach et al. (2002). The three radio halo emission models (A, B, C) described by Eqs. (5-7) are displayed in comparison to the isotropised synchrotron kernel (see text). The SZ decrement (a negative flux) is also shown. The solid angle over which the observed fluxes were integrated may vary from observation to observation. For the theoretical models, the flux from the full cluster volume up to the assumed position of the accretion shock is given.

the Compton $y$-parameter. $n_{\mathrm{e}}$, and $T_{\mathrm{e}}$ are the electron density and temperature, respectively, $l$ is the coordinate along the line-of-sight, and

$g(x)=\frac{x^{4} \mathrm{e}^{x}}{\left(\mathrm{e}^{x}-1\right)^{2}}\left(x \frac{\mathrm{e}^{x}+1}{\mathrm{e}^{x}-1}-4\right) \approx-2 x^{2}$ for $x \ll 1$,

describes the spectral distortions ${ }^{1}$.

The area integrated SZ distortion of the CMB by a galaxy cluster can be described as a SZ-flux (even if this is negative in the Rayleigh-Jeans part of the CMB):

$F_{\mathrm{SZ}}(v)=\frac{2 \sigma_{\mathrm{T}} i_{0} E_{\mathrm{th}, \mathrm{e}}}{3 m_{\mathrm{e}} c^{2} D^{2}} g(x)$,

where $E_{\mathrm{th}, \mathrm{e}}$ is the total energy of all thermal cluster electrons, and $D$ is the angular cluster distance.

In our examples, we assume the cluster to be isothermal, spherically symmetric, and its electron density well described by a beta-model $n_{\mathrm{e}}(r)=n_{\mathrm{e}, 0}\left[1+\left(r / r_{\mathrm{c}}\right)^{2}\right]^{-3 \beta / 2}$ up to the accretion shock wave located at radius $r=R$. For Coma, we adopt $k T_{\mathrm{e}}=8.2 \mathrm{keV}, n_{\mathrm{e}, 0}=3 \times 10^{-3} h_{50}^{1 / 2} \mathrm{~cm}^{-3}$, $r_{\mathrm{c}}=0.4 h_{50}^{-1} \mathrm{Mpc} \hat{=} 10^{\prime}, \beta=0.8$ (Briel et al. 1992), $R=$ $5 h_{50}^{-1} \mathrm{Mpc}$ (Enßlin et al. 1998), and $D=140 h_{50}^{-1} \mathrm{Mpc}$. This leads to $E_{\mathrm{th}, \mathrm{e}}^{\mathrm{Coma}}=7.7 \times 10^{63} h_{50}^{-5 / 2} \mathrm{erg}$, and a SZ-luminosity of

$F_{\mathrm{SZ}}^{\mathrm{Coma}}(v)=3.0 g(x) h_{50}^{-\frac{1}{2}} \mathrm{Jy} \approx-1.9 \times 10^{-3} v_{\mathrm{GHz}}^{2} h_{50}^{-\frac{1}{2}} \mathrm{Jy}$,

${ }^{1}$ In the case of hot clusters $(\sim 10 \mathrm{keV})$ relativistic effects modify the shape of $g(x)$. However, the corrections are negligible on the RayleighJeans side of the CMB spectrum for $k T_{\mathrm{e}} \ll \mathrm{MeV}$ (e.g. see Fig. 6 in Enßlin \& Kaiser 2000), so that Eq. (2) is an accurate estimate in our frequency regime $(x \ll 1)$. The relativistic electron population in Coma is also not able to modify this behaviour significantly (Enßlin \& Kaiser 2000; Blasi et al. 2000; Shimon \& Rephaeli 2002).

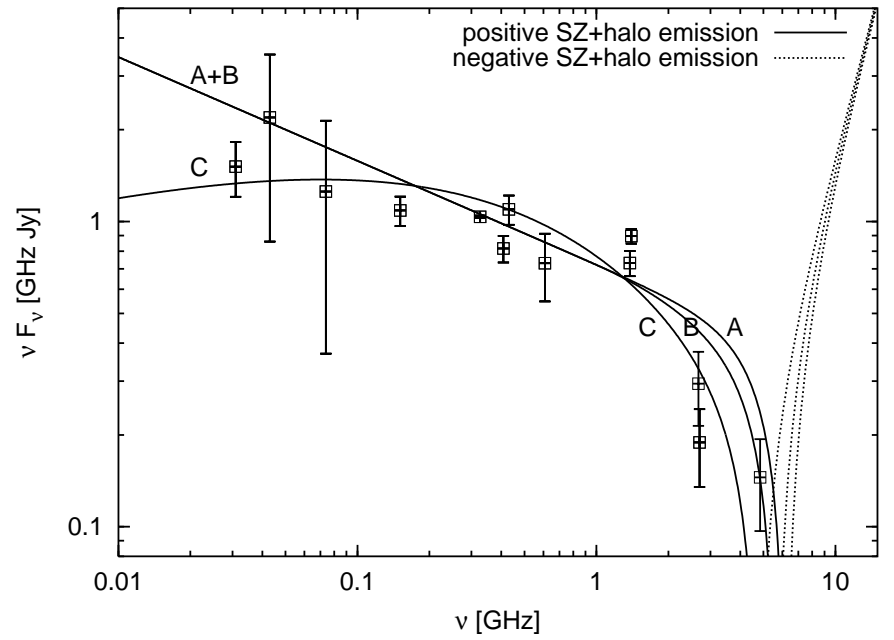

Fig. 2. Radio spectrum of the Coma cluster in $v F(v)$ for better display (see Fig. 1). Solid (dotted) curves represent the total cluster emission (CMB decrement) of the three halo emission models (A, B, C) combined with the SZ decrement.

where $v_{\mathrm{GHz}}=v / \mathrm{GHz}$. The same parameters give a central $y$-parameter of $y_{\text {model }}^{\text {Coma }}=0.85 \times 10^{-4} h_{50}^{-1 / 2}$, which is in good agreement with the measured value of $y_{\mathrm{obs}}^{\mathrm{Coma}}=(0.7 \ldots 1.0) \times 10^{-4}$ (Herbig et al. 1995; Mason et al. 2001; De Petris et al. 2002).

\section{Coma radio halo}

Cluster radio halos have steep radio spectra $\left(F_{\text {halo }} \propto v^{-s}\right)$ with spectral indexes $s>1$. In the case of Coma, there seems to be a further steepening beyond $1.4 \mathrm{GHz}$. The current data as compiled by Thierbach et al. (2002) is displayed in Fig. 1 in comparison with three model spectra.

A) A steep power law spectrum:

$F_{\text {halo, } 1}^{\text {Coma }}(v)=0.723 v_{\mathrm{GHz}}^{-1.34} \mathrm{Jy}$.

B) As A) but with a soft high frequency steepening:

$F_{\text {halo, } 2}^{\text {Coma }}(v)=0.723 v_{\mathrm{GHz}}^{-1.34-0.1 \mathrm{max}\left(0, \ln v_{\mathrm{GHz}}\right)} \mathrm{Jy}$.

C) A flat power law spectrum with exponential cutoff:

$F_{\text {halo }, 3}^{\text {Coma }}(v)=3.42 v_{\mathrm{GHz}}^{-0.8} \exp \left(-\sqrt{v_{\mathrm{GHz}}}\right) \mathrm{Jy}$.

The last spectrum is a fit to the data by Thierbach et al. (2002), following a functional form proposed by Schlickeiser et al. (1987) as a model for a continously in-situ accelerated electron population with exponential high energy cutoff. It should be noted that the functional form of Eq. (7) was estimated with the monochromatic approximation for the synchrotron emissivity of monoenergetic electrons. With the correct calculations, therefore, the high frequency cutoff would be much smoother ${ }^{2}$. In order to get a similar sharp cutoff in the emission spectrum, a sharp cutoff in the electron spectrum is required, as e.g. in the time dependent in-situ model investigated by

2 The synchrotron spectrum of a monoenergetic electron population in an isotropic distribution of magnetic fields is displayed in Fig. 1. A synchrotron spectrum can not be more strongly bent than this emission spectrum kernel. 


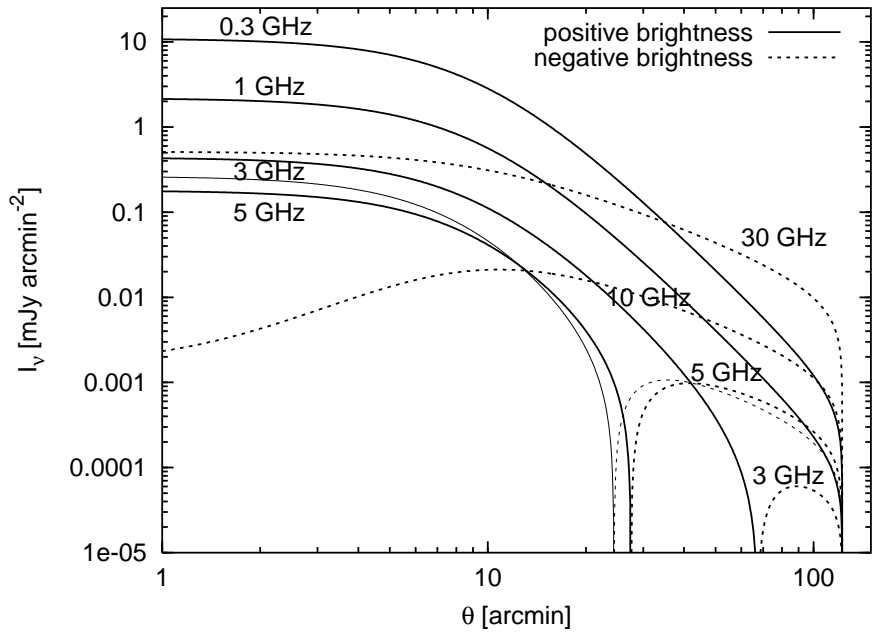

Fig. 3. Radial brightness profile of our toy-model of the Coma cluster at different frequencies as it results from combining the radio halo emission and the SZ decrement profiles. The total emissivity follows the curve of model B displayed in Fig. 2. At $0.3 \mathrm{GHz}(30 \mathrm{GHz})$ the practically unmodified radio halo (SZ decrement) profile is visible. The thin line gives the $5 \mathrm{GHz}$ model for $\alpha=1.1, \beta=0.85$, illustrating the strong dependence of the profiles on the parameters.

Brunetti et al. (2001). Emission model C reproduces the observed data only if the SZ decrement is neglected (see Fig. 2). However, as explained above, this is not a problem for the continuous in-situ model, since correctly estimated, it produces a softer cutoff than given by model $\mathrm{C}$. In model $\mathrm{C}$ the SZeffect would dominate above $4.7 \mathrm{GHz}$ and lead to a decrement at the observed frequency of $4.9 \mathrm{GHz}$ in contrast to the data. In model $\mathrm{B}$ (A) the change of sign of the total radio fluxes is at $5.7 \mathrm{GHz}(6.2 \mathrm{GHz})$. Model A predicts too much flux at high frequencies, but model B seems to be consistent with the data. Thus, only a weak spectral steepening of the radio halo emission above $1 \mathrm{GHz}$ is required by the data.

Such a weak steepening can also be explained in the secondary electron model. There the radio halo spectrum steepening may occur due to a steepening of the parent cosmic ray proton spectrum.

\section{Radial brightness profiles}

The various radio emission processes of galaxy clusters can partly be separated by their morphological difference. The SZ-emission has a spatially broad brightness distribution, since it is proportional (in an isothermal model) to the line-of-sight projected electron density. The radio emission seems to be as concentrated as the Bremsstrahlung X-ray emission of the thermal cluster gas: $I_{\text {halo }} \propto I_{\mathrm{X}}^{\alpha}$, where $\alpha \approx 1$ (Govoni et al. 2001). Since the X-ray emissivity is proportional to $n_{\mathrm{e}}^{2}$, we assume the radio halo emissivity to exhibit the same scaling. Note, that secondary electron models would usually predict a steeper scaling ( $\alpha \approx 1.3$ by Dolag $\&$ Enßlin 2000, but see Miniati et al. 2001), which therefore seem to be disfavoured (Govoni et al. 2001; Brunetti 2002).

In Fig. 3 we used a toy model to illustrate the consequences of the SZ decrement on the properties of the radio brightness profiles as a function of frequency. For that purpose we assume a uniform emission spectrum given by model B (Eq. (6)) and the above mentioned radial scaling ${ }^{3}$. At low ( $\lesssim \mathrm{GHz}$ ) frequencies the radio halo emission dominates. With increasing frequency a negative SZ-bowl surrounding the residual of the central visible radio halo emission appears and grows (dotted line in Fig. 3). At $10 \mathrm{GHz}$ the SZ decrement dominates at every angular position, but its central part still reveals the presence of the radio halo emission by exhibiting a dip in the SZ decrement. At $30 \mathrm{GHz}$ the radio halo emission is insignificant, so that this frequency can safely be used for SZ-measurements.

Even at frequencies, at which the total radio emission is zero or negative ( $\gtrsim 5 \mathrm{GHz}$ ), a central positive emission region can exist if the frequency is not too high $(<10 \mathrm{GHz})$. Thus, a positive flux may be attributed to such a radio map, if only the region with positive flux is included into the map analysis. Especially, since the negative bowl around the central region is relatively shallow (it dominates only the total flux due to its larger area) there is always the danger that it is regarded as noise, or as a remnant of imperfect map reconstruction in the case of an interferometric measurement without zero-spacing flux. In the case that the radio halo luminosity of a cluster is assumed to be equal to the flux within the positive region, the real halo luminosity would be underestimated (e.g. by $34 \%$ at $5 \mathrm{GHz}$ in our toy-model).

In the frequency range of several $\mathrm{GHz}$, there exist a relatively sharp outer edge of the positive brightness region, which (in our toy-model) is located at $\theta \approx 70^{\prime}$ for $3 \mathrm{GHz}$, and at $\theta \approx 30^{\prime}$ for $5 \mathrm{GHz}$, whereas the synchrotron emission extends up to $\theta=120^{\prime}$. The exact location of the edge of the positive emission region depends sensitively on several of the model parameters $\left(r_{\mathrm{c}}, \beta, \alpha\right.$, see Fig. 3) and on here not modelled details of the radio halo emission spectrum as a function of cluster radius. However, a similar frequency trend of the edge of positive emission can be seen by visual inspection of the Coma radio maps at $1.4 \mathrm{GHz}$ (Deiss et al. 1997) which exhibits positive emission up to $\theta \gtrsim 45^{\prime}$, and the maps at 2.7 and $4.9 \mathrm{GHz}$ (Thierbach et al. 2002) which seem to be positive only up to $\theta \approx 20^{\prime}$ and $\theta \approx 10^{\prime}$, respectively. Although there is likely a contribution to this shrinking due to spectral steepening at larger radii, our estimate and the actual SZ measurements argue that a significant fraction of the emission area shrinking is due to the unavoidable SZ-effect. The exact amount is difficult to estimate due to our still limited knowledge of the exact radio halo emission profile. However, the prediction of the SZ-decrement profile is quiet robust for the central cluster regions due to the observationally well constrained $y$-parameter with an error of only $\sim 10 \%$. Therefore, it is possible to state that for the measurements of Thierbach et al. (2002) at $2.7 \mathrm{GHz}$ and $4.9 \mathrm{GHz}$ the central SZ-decrement is of the order of $30 \%$ and $50 \%$ of the $1-\sigma$ noise level (per beam as shown in their Figs. 2 and 3), respectively. Since the SZ decrement strength decreases radially only slowly, it should affect the outer regions

\footnotetext{
3 The resulting spatially constant spectral index at low $(<\mathrm{GHz})$ frequencies is not exactly what is observed since a radial spectral steepening between 0.3 and $1.4 \mathrm{GHz}$ is found by (Giovannini et al. 1993).
} 
of the rapidly decreasing radio halo ${ }^{4}$. Thus it should be statistically detectable there due to the sufficient large number of resolution elements covering the cluster outskirts. A careful analysis of high signal-to-noise high frequency maps of radio halos are expected to reveal an extended decrement at outer cluster regions.

\section{Conclusion}

Measurements of cluster radio halos at several $\mathrm{GHz}$ are contaminated by the thermal SZ effect, or vice versa, at $\sim 10 \mathrm{GHz}$, measurements of the SZ effect have to take the possible presence of cluster radio halos into account. The morphology of the radio emission of a galaxy cluster at such frequencies, where the total radio halo emission and the total SZ decrement roughly compensate each other, is that of a central emission region surrounded by a shallow negative bowl. This complex morphology can lead to ambiguities in observational determinations of radio halo fluxes, since the result depends on the adopted procedure (flux from the full cluster area, or only flux from regions of positive brightness). We therefore stress the need to communicate up to which radius a cluster radio halo flux was integrated. It would be best, if such measurements would be provided for several radii. Radio halo emission measurements should carefully be corrected for the unavoidable SZ decrement (see also Liang et al. 2000).

Using the Coma cluster as an example we demonstrated that the existing radio maps at 2.7 and $4.9 \mathrm{GHz}$ of the Coma radio halo should be affected by the $\mathrm{SZ}$ decrement. This is very likely part of the reason (in combination with radial spectral steepening of radio halo emissivity and a decreasing $S / N$ ratio) why the apparent radio halo size decreases with increasing frequency. The observed sharp spectral steepening of the total radio flux of Coma seems to be partly - but not completely due to the SZ effect. This implies that only a weakly bended emission spectrum is required to explain the data, and consequently the constrain arising from the apparent strong spectral steepening on some proposed radio halo formation scenarios as continuous in-situ acceleration and secondary electron injection is relaxed.
Acknowledgements. I acknowledge several useful comments by E. Churazov, K. Jedamzik, F. Miniati, and C. Vogt. This work was done in the framework of the EC Research and Training Network The Cosmic Microwave Background.

\section{References}

Böhm, C., Enßlin, T. A., \& Silk, J. 2002 [astro-ph/0208458]

Blasi, P., Olinto, A. V., \& Stebbins, A. 2000, ApJ, 535, L71

Briel, U. G., Henry, J. P., \& Böhringer, H. 1992, A\&A, 259, L31

Brunetti, G. 2002, in Matter and Energy in Clusters of Galaxies, ed. S. Bowyer, \& C.-Y. Hwang, ASP Conf. Series, in preparation [astro-ph/0208074]

Brunetti, G., Setti, G., Feretti, L., \& Giovannini, G. 2001, MNRAS, 320,365

Buote, D. A. 2001, ApJ, 553, L15

Colafrancesco, S., \& Mele, B. 2001, ApJ, 562, 24

De Petris, M., D’Alba, L., Lamagna, L., et al. 2002, ApJ, 574, L119

Deiss, B. M., Reich, W., Lesch, H., \& Wielebinski, R. 1997, A\&A, 321, 55

Dennison, B. 1980, ApJ, 239, L93

Dolag, K., \& Enßlin, T. A. 2000, A\&A, 362, 151

Enßlin, T. A., Biermann, P. L., Klein, U., \& Kohle, S. 1998, A\&A, 332,395

Enßlin, T. A., \& Kaiser, C. R. 2000, A\&A, 360, 417

Enßlin, T. A., \& Röttgering, H. 2002, A\&A, 396, 83

Giovannini, G., \& Feretti, L. 2000, New Astron., 5, 335

Giovannini, G., Feretti, L., Venturi, T., Kim, K. T., \& Kronberg, P. P. 1993, ApJ, 406, 399

Giovannini, G., Tordi, M., \& Feretti, L. 1999, New Astron., 4, 141

Govoni, F., Enßlin, T. A., Feretti, L., \& Giovannini, G. 2001, A\&A, 369,441

Herbig, T., Lawrence, C. R., Readhead, A. C. S., \& Gulkis, S. 1995, ApJ, 449, L5

Jaffe, W. J. 1977, ApJ, 212, 1

Kempner, J. C., \& Sarazin, C. L. 2001, ApJ, 548, 639

Liang, H., Hunstead, R. W., Birkinshaw, M., \& Andreani, P. 2000, ApJ, 544, 686

Mason, B. S., Myers, S. T., \& Readhead, A. C. S. 2001, ApJ, 555, L11

Miniati, F., Jones, T. W., Kang, H., \& Ryu, D. 2001, ApJ, 562, 233

Schlickeiser, R., Sievers, A., \& Thiemann, H. 1987, A\&A, 182, 21

Shimon, M., \& Rephaeli, Y. 2002, ApJ, 575, 12

Sunyaev, R. A., \& Zeldovich, I. B. 1980, ARA\&A, 18, 537

Thierbach, M., Klein, U., \& Wielebinski, R. 2002, A\&A, in press [astro-ph/0210147]

Zeldovich, Y. B., \& Sunyaev, R. A. 1969, Ap\&SS, 4, 301

${ }^{4}$ E.g. at the location of the 3- $\sigma$ brightness contour, located at $10^{\prime}$ $\left(7^{\prime}\right)$ in the $2.7 \mathrm{GHz}(4.9 \mathrm{GHz})$ map of Thierbach et al. (2002), with a brightness of $0.038(0.067) \mathrm{mJy} / \mathrm{arcmin}^{2}$, the negative SZ contribution is $-0.0026(-0.011) \mathrm{mJy} / \operatorname{arcmin}^{2}$. 